\title{
Efeitos do treino funcional de membro superior em condição de dupla tarefa na doença de Parkinson
}

\author{
Effects of upper limb functional training in the dual task condition in Parkinson's disease
}

\author{
Caroline Kaori Tomo ${ }^{1}$, Vanessa Silva Pereira², Sandra Maria Alvarenga Anti \\ Pompeu ${ }^{3}$, José Eduardo Pompeu 4
}

\section{RESUMO}

Objetivo. Comparar os efeitos de duas condições de treinamento, tarefa simples (TS) versus dupla tarefa (DT), nas funçóes do membro superior de pacientes com doença de Parkinson (PDP). Método. 14 PDP foram randomizados entre os grupos controle (GC, n=7) e experimental (GE, n=7) e avaliados antes e após o treinamento pelo mesmo avaliador cego. O desfecho primário foi o teste Box \& Block $(\mathrm{B} \& \mathrm{~B})$ e o secundário o número de repetiçóes de quatro tarefas funcionais nas condiçōes de TS e DT: vestir um casaco, pentear cabelo, atender ao telefone e levar um copo à boca. Foram realizadas cinco sessóes de uma hora de treinamento, nas quais os pacientes praticavam cada tarefa por 15 minutos: o GC em TS e o GE de modo associado a outra tarefa de fluência verbal (DT). Resultados. ambos os grupos apresentaram melhora no B\&B; no pentear cabelo, o GC melhorou em DT. Na tarefa de atender ao telefone, o GC melhorou o desempenho em TS, enquanto que o GE melhorou em DT. Conclusáo. os treinamentos contribuíram para a melhora da função do membro superior de PDP. A condição de treinamento e as características das tarefas podem interferir na melhora do desempenho.

Unitermos. Doença de Parkinson, Atenção, Função Executiva, Membro Superior

Citaçáo. Tomo CK, Pereira VS, Pompeu SMAA, Pompeu JE. Efeitos do treino funcional de membro superior em condiçáo de dupla tarefa na doença de Parkinson.

\begin{abstract}
Objective. to compare the effects of two conditions of training, single task (ST) versus dual task (DT), on upper limb functions of patients with Parkinson's disease (PPD). Method. 14 PPD were randomized between control group (CG) and experimental group (EG), seven in each. Patients were assessed before and after training by the same blinded evaluator. The primary outcome was the Box \& Block test $(B \& B)$ and the secondary outcome was the number of repetition of four functional tasks of upper limb: put on a coat, comb hair, answer a phone and take a glass to mouth. It were performed five sessions of training of one hour, in which patients practiced 15 minutes each task: CG in ST and EG associated with other task of verbal fluency (DT). Results. both groups showed performance improvement on $\mathrm{B} \& \mathrm{~B}$; CG improved the task of comb hair in DT and answer a phone in ST, while the EG improved the task of answer a phone and in DT. Conclusion. both training contributed to the upper limb function improvement of PPD. The condition of training and the characteristics of the tasks can influence on the performance improvement.
\end{abstract}

Keywords. Parkinson's Disease, Attention, Executive Function, Upper Limb

Citation. Tomo CK, Pereira VS, Pompeu SMAA, Pompeu JE. Effects of upper limb functional training in the dual task condition in Parkinson's disease.

1. Fisioterapeuta, Residente na UNIFESP, São Paulo-SP, Brasil.

2. Fisioterapeuta, Pós-graduanda no Centro de estudos de terapias manuais, São Paulo-SP, Brasil

3. Fisioterapeuta, Mestre, Professora adjunta da Universidade Paulista, São Paulo-SP, Brasil

4. Fisioterapeuta, Doutor, Pós-doutorando na UNIFESP, Professor da Universidade de São Paulo, São Paulo-SP, Brasil 


\section{INTRODUÇÃO}

A execução eficiente de atividades cotidianas compreende a capacidade de realizar mais de uma ação simultaneamente em diversas situaçôes, o que constitui um pré-requisito para uma vida funcional, independente e segura.

A habilidade de dividir a atenção em diferentes açôes concomitantes é denominada de desempenho simultâneo ou dupla tarefa (DT), condição especialmente desafiadora para pacientes com doença de Parkinson (PDP) ${ }^{1}$.

A dificuldade apresentada por PDP no desempenho de DT ainda não é totalmente compreendida, porém hipóteses são levantadas, incluindo déficits de função executiva e limitação de recursos atentivos disponíveis para a execução de ações concorrentes ${ }^{2,3}$, dificuldades em compartilhar recursos atentivos entre duas tarefas ${ }^{2,4}$, além da perda de automaticidade decorrente da lesão dos núcleos da base e circuitos associados 5 .

Prejuízos na execução da marcha ${ }^{6,7}$ e na manutenção do equilíbrio ${ }^{8}$ em DT são amplamente descritos, porém atividades envolvendo o membro superior, como escrever e ao mesmo tempo falar ao telefone ou retirar uma carteira do bolso enquanto conversa com um vendedor, são tão difíceis ou até mesmo impossíveis para PDP dependendo do estágio da doença ${ }^{9,10}$.

Movimentos como os de alcançar e manipular objetos são componentes críticos para atividades de vida diária, e limitaçóes no desempenho destas habilidades pode afetar a independência funcional. A bradicinesia, considerada como um dos sinais clínicos mais incapacitantes na DP, é caracterizada pela lentidão na execução e dificuldade para iniciar movimentos voluntários. Esta alteração é muito evidente em situações que requerem controle motor fino, como abotoar e utilizar utensílios. A bradicinesia piora em condiçôes de divisão de atenção ou de desempenho de duas ou mais tarefas simultâneas ${ }^{11}$.

A manipulação de objetos é considerada um processo que requer alta demanda atentiva em PDP, devido à perda de automaticidade e dependência de realimentaçáo visual. O desvio da atenção da ação em curso promove a diminuição na velocidade e na amplitude de movimento, mostrando a dependência de um foco atentivo na DP. Isto explicaria a dificuldade de realizar atividades manu- ais em DT, onde a atenção deve ser deslocada ou compartilhada com uma tarefa concorrente11. De fato, PDP apresentam prejuízos na execução de DT envolvendo o membro superior tanto na combinação de duas açóes motoras ${ }^{12,13}$ como na associação com uma tarefa cognitiva ${ }^{9-11,14,15}$, indicando que não se trata de uma alteração puramente motora ${ }^{16}$.

Estratégias de tratamento baseadas na prática em condições de DT têm sido propostas no sentido de promover a melhora na funcionalidade de PDP. Dois estu$\operatorname{dos}^{17,18}$ verificaram que o treinamento em DT promoveu aumento do tamanho do passo e da velocidade da marcha além de reduzir a sua variabilidade. Outro estudo analisou os efeitos do treino em DT na manutenção da postura bípede durante a leitura de sentenças em jovens, idosos e PDP, porém apenas os jovens apresentaram melhora após o treinamento ${ }^{19}$.

Em relação ao membro superior, apenas um estudo analisou o desempenho de idosos e PDP no ato de vestir uma camisa em TS e DT (associado a uma tarefa verbal) após três repetiçóes de cada uma das condiçôes. Foi verificado aumento da velocidade de execução e diminuição do número de erros na terceira repetição em ambos os grupos $^{14}$. No entanto, este estudo analisou somente uma tarefa funcional e o número de repetiçóes utilizado no protocolo de intervenção foi pequeno. Com isso, os resultados não podem ser generalizados para outras tarefas funcionais de membro superior.

Deste modo, há poucas evidências de que PDP podem apresentar melhora de desempenho das funçóes de membro superior quando treinados em DT. Além disso, ainda não há evidências de que o treinamento em DT seja superior ao realizado em TS. Assim, o objetivo do presente estudo foi comparar os efeitos de duas condições de treinamento (TS versus DT) em quatro diferentes funções do membro superior em PDP no sentido de se verificar qual seria a melhor estratégia de intervenção.

\section{MÉTODO}

\section{Tipo de estudo}

Ensaio clínico randomizado cego.

\section{Amostra}

Foram selecionados pacientes com diagnóstico clí- 
nico de DP idiopática medicados com levodopa ou seus sinergistas; idade entre 65 e 75 anos; nos estágios 1 e 2 da escala de Hoehn e $\mathrm{Yahr}^{20}$, com boa acuidade visual e auditiva, sem outras doenças neurológicas ou ortopédicas, ausência de demência (avaliada através do Mini-Exame de Estado $\mathrm{Mental}^{21}$ com pontuação mínima de 23) ou depressão (de acordo com a Escala de Depressão Geriátrica-1522, pontuação maior que cinco). Cada paciente foi informado sobre as características da intervenção e assinou Termo de Consentimento Livre e Esclarecido antes do início do estudo. Este estudo foi aprovado pelo Comitê de Ética e Pesquisa do Centro Universitário São Camilo (número 262.928).

\section{Local do estudo}

O estudo foi conduzido na Associação Brasil Parkinson, São Paulo, Brasil, em agosto de 2013.

\section{Procedimento \\ Randomização}

Os nomes dos participantes foram escritos e colocados dentro de envelopes selados, sendo separados aleatoriamente entre grupo controle (GC) e grupo experimental (GE) por uma das pesquisadoras (Figura 1). Os pacientes foram divididos em dois grupos de forma randomizada, sete pacientes em cada grupo.

\section{Intervenção}

Foram realizadas cinco sessóes de treinamento com duração de uma hora cada, realizadas em dias consecutivos. Cada tarefa funcional foi praticada por 15 minutos em cada sessão. O GC realizou o treinamento apenas em condição de TS, enquanto o GE treinou em DT.

\section{Treinamento do GC em TS}

O GC realizou o treinamento em TS, ou seja, com a atenção focada no ato motor e sem a realização de nenhuma outra tarefa concorrente. Foram treinadas quatro tarefas motoras funcionais: (1) vestir um casaco (apanhar, vestir e abotoar o casaco), (2) pentear cabelo (segurar um pente e pentear os cabelos no sentido fronto-occipital), (3) atender ao telefone (pegar o telefone no suporte e leva-lo até a orelha) e (4) levar um copo até a boca (pegar o copo em uma mesa e leva-lo até a boca). Estas tarefas foram selecionadas devido à sua relevância funcional e ao seu frequente desempenho nas atividades de vida diária. Somente a tarefa de vestir um casaco foi realizada em pé, enquanto as demais foram treinadas na posição sentada. Os pacientes foram orientados a realizar o maior número possível de repetiçóes de cada tarefa durante 15 minutos, quando passavam para a execução da próxima tarefa. Entre uma tarefa e outra, os pacientes descansavam por um minuto. A ordem na qual as tarefas funcionais foram treinadas foi pseudo-randomizada.

\section{Treinamento do GE em DT}

O GE realizou o treinamento das tarefas funcionais de modo concomitante à execução de uma atividade de fluência verbal na qual deveria falar nomes próprios iniciados com determinada letra. Não houve orientaçóes em relação à priorização das tarefas, ou seja, o paciente não foi orientado para priorizar o desempenho da tarefa funcional ou da fluência verbal.

\section{Avaliaçôes}

Os dois grupos foram avaliados antes e após o treinamento, pelo mesmo avaliador cego. A avaliação foi realizada por um pesquisador que náo tinha conhecimento sobre qual dos grupos os pacientes faziam parte.

\section{Desfecho primário}

O desfecho primário do estudo foi o Teste de Caixa de Blocos (Box \& Block test - B\&B), no qual o indivíduo deve transportar pequenos blocos de madeira de um lado para outro em uma caixa bipartida; o tempo do teste é de 60 segundos, sendo registrado o número de blocos colocados no lado oposto da caixa. O teste foi realizado com a mão dominante ${ }^{23}$.

Desfecho secundário

O desfecho secundário foi o desempenho dos grupos nas quatro tarefas motoras funcionais. Foi registrado o número de repetições executadas de cada tarefa em 30 segundos em duas condiçóes: (1) em TS, ou seja, realizando somente a tarefa funcional e (2) em DT, associando o desempenho da tarefa funcional a uma tarefa de fluência verbal (recitar nomes próprios com determinada letra pré-estabelecida). 
Figura 1. Fluxograma do estudo:14 pacientes, divididos em dois grupos.

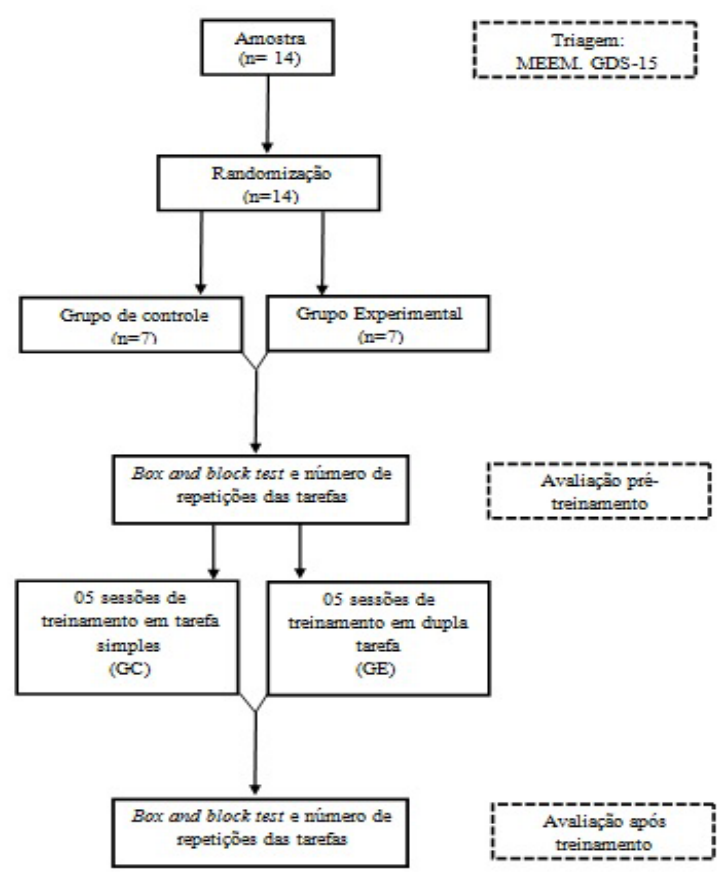

\section{Análise estatística}

Para analisar os efeitos do treinamento sobre o desempenho das tarefas funcionais nas duas condiçóes de avaliação (TS e DT) e para a comparação do desempenho entre os grupos GC e GE, após testes de normalidade (teste de Kolmogorov-Smirnov) e homocedasticidade (teste de Levene) e análise de distribuição de resíduos, foram realizadas quatro análises de variância de medidas repetidas (RM-ANOVA 2X2). Para a avaliação do desempenho no B\&B foi realizada outra RM-ANOVA 2x2 (dois grupos em duas condiçóes, TS e DT).

Para os efeitos que alcançaram nível de significância, foi realizado o Teste Pós Hoc de Tukey para a verificação de eventuais diferenças entre os mesmos. Para toda a análise o nível de significância adotado foi de 5\%.

\section{RESULTADOS}

Participaram do estudo 14 pacientes com DP idiopática nos estágios 1 e 2 da escala Hoehn e Yahr, 12 do gênero masculino e dois do feminino (Tabela 1 ). A média de idade dos pacientes do GC foi $67,3 \pm 3,1$ anos e do GE foi 70,7 $\pm 4,2$ anos. Todos os participantes completaram o treinamento sem quaisquer efeitos adversos como náuse- as, fadiga, tontura ou qualquer outro tipo de queixa.

A Tabela 2 resume os dados referentes ao desempenho do GC e GE nas quatro tarefas motoras primárias e no teste $\mathrm{B} \& \mathrm{~B}$ antes e após o treinamento

Ambos os grupos apresentaram melhora no B\&B após o treinamento. Não houve diferença entre os grupos. O GC apresentou melhora no desempenho da tarefa de pentear cabelo na condição de DT $(\mathrm{p}<0,05)$. Na tarefa de atender ao telefone, o GC melhorou o desempenho na condição de TS $(\mathrm{p}<0,05)$ e o GE na condição de DT $(\mathrm{p}<0,05)$. Ambos os grupos não apresentaram melhora do desempenho nas tarefas de vestir o casaco e de levar o copo à boca.

\section{DISCUSSÂO}

As hipóteses do presente estudo foram: (1) ambos os treinamentos promoveriam melhora no desempenho no B\&B; (2) o GC apresentaria melhor desempenho em condição de TS sem efeito sobre a condição de DT e (3) o treinamento em DT poderia contribuir para a melhora do desempenho tanto em DT quanto em TS. Os resultados confirmaram a hipótese de que ambos os grupos melhorariam o desempenho no B\&B após o treinamento. Entretanto, em relação às atividades funcionais, o GC apresentou melhora no desempenho da tarefa de pentear cabelo em condiçấo de DT e na tarefa de atender ao telefone em TS. Enquanto que o GE apresentou melhora na tarefa de atender ao telefone em DT. Ambos os grupos não apresentaram melhora no desempenho das outras atividades tanto em TS quanto em DT.

A melhora no desempenho do B\&B pode ter ocor-

Tabela 1. Dados demográficos e características clínicas dos pacientes.

\begin{tabular}{lccc}
\hline $\begin{array}{l}\text { Características dos } \\
\text { Pacientes }\end{array}$ & GC & GE & $\begin{array}{c}\text { Valor } \\
\text { de } \mathbf{p}\end{array}$ \\
\hline Idade & $67,3 \pm 3,1$ & $70,7 \pm 4,2$ & 0,06 \\
Hoehn e Yahr & $1,7 \pm 0,5$ & $1,6 \pm 0,5$ & 0,36 \\
Gênero & $\mathrm{M}=6$ e F=1 & $\mathrm{M}=6$ e F=1 & -- \\
MEEM & $25,6 \pm 2,7$ & $27,0 \pm 1,9$ & 0,34 \\
GDS-15 & $6,1 \pm 0,9$ & $6,4 \pm 1,4$ & 0,67 \\
\hline
\end{tabular}

GC=grupo controle; GE=grupo experimental; MEEM=Mini Exame do Estado Mental; GDS-15=Escala de Depressão Geriátrica-15; M=masculino; $\mathrm{F}=$ feminino, 
Tabela 2. Valores da média e desvio padrão referentes ao número de repetiçóes executadas nas quatro tarefas motoras primárias e no teste Bひ̉ $B$, pré e pós treinamento, no grupo controle $(G C)$ e grupo experimental $(G E)$.

\begin{tabular}{|c|c|c|c|c|c|c|c|c|}
\hline & \multicolumn{4}{|c|}{ GC } & \multicolumn{4}{|c|}{ GE } \\
\hline & \multicolumn{2}{|c|}{ Pré treinamento } & \multicolumn{2}{|c|}{ Pós treinamento } & \multicolumn{2}{|c|}{ Pré treinamento } & \multicolumn{2}{|c|}{ Pós treinamento } \\
\hline & TS & DT & TS & DT & TS & DT & TS & DT \\
\hline $\mathrm{B} \& \mathrm{~B}$ & \multicolumn{2}{|c|}{$45,3 \pm 15,2$} & \multicolumn{2}{|c|}{$59,0 \pm 11,5^{*}$} & \multicolumn{2}{|c|}{$45,6 \pm 10,7$} & \multicolumn{2}{|c|}{$52,0 \pm 11,1^{*}$} \\
\hline Vestir o casaco & $1,9 \pm 0,4$ & $1,0 \pm 0,8$ & $2,7 \pm 2,0$ & $2,6 \pm 2,1$ & $2,1 \pm 1,7$ & $1,3 \pm 1,0$ & $2,6 \pm 1,7$ & $2,1 \pm 2,3$ \\
\hline Pentear cabelo & $24,0 \pm 8,2$ & $13,6 \pm 7,7$ & $34,7 \pm 16,2$ & $29,3 \pm 13,7^{*}$ & $20,1 \pm 6,5$ & $11,7 \pm 7,3$ & $25,1 \pm 9,0$ & $20,1 \pm 6,9$ \\
\hline Atender um telefone & $10,7 \pm 3,7$ & $7,9 \pm 1,8$ & $24,6 \pm 9,1^{*}$ & $18,3 \pm 7,7$ & $13,4 \pm 4,9$ & $7,6 \pm 1,9$ & $18,3 \pm 4,6$ & $15,3 \pm 3,8^{*}$ \\
\hline Copo à boca & $16,9 \pm 6,0$ & $9,4 \pm 4,1$ & $31,9 \pm 11,9$ & $26,7 \pm 11,9$ & $20,0 \pm 8,5$ & $11,6 \pm 5,9$ & $26,6 \pm 8,6$ & $20,3 \pm 5,6$ \\
\hline
\end{tabular}

$\mathrm{GC}=$ grupo controle; $\mathrm{GE}=$ grupo experimental; $\mathrm{TS}=$ tarefa simples; $\mathrm{DT}=$ dupla tarefa; $\mathrm{B} \& \mathrm{~B}=$ Box and block test; ${ }^{\mathrm{p}}<0,05$.

rido devido ao intenso treinamento de tarefas motoras relacionadas com o alcance, preensão, levantamento, deslocamento e desprendimento de objetos, demandas motoras exigidas no teste. Deste modo, acredita-se que tais habilidades possam ter sido transferidas do treinamento para o desempenho no teste. Como a demanda motora dos treinamentos era semelhante para os dois grupos, acredita-se que a repetição dos padróes de movimento necessários para o desempenho das tarefas funcionais tenha contribuído para a melhora no $\mathrm{B} \& \mathrm{~B}$ em ambos os grupos.

A prática de uma tarefa que contém um "padrão fundamental de movimento" favorece a transferência de aprendizagem para outras tarefas que utilizam aquele padrão; a transferência é um fenômeno que ocorre quando representações de movimento previamente adquiridas facilitam a execução de movimentos semelhantes, ou seja, a experiência prévia facilita o desempenho de açóes subsequentes ${ }^{24}$. O B\&B, mesmo não treinado, pode ter sido facilitado pelo treinamento de movimentos semelhantes no mesmo membro.

O resultado obtido pelo GC na TS de atender ao telefone era esperado e provavelmente associado ao treinamento específico realizado; a atenção focada para a ação, sem distraidores, pode ter contribuído para o incremento do desempenho.

Já o melhor desempenho do GC em DT não era um resultado esperado, uma vez que foi treinada apenas de forma isolada. Devido à lesão nos núcleos da base, pacientes com DP apresentam dificuldade em realizar ações previamente aprendidas, porém ainda tem potencial para aprendizado motor ${ }^{25}$. O treino em TS realizado pelo GC pode ter promovido a automaticidade, liberando áreas corticais envolvidas com o direcionamento da atenção para a ação, para a execução de uma tarefa secundária concorrente ${ }^{26}$. Os movimentos automáticos são controlados pelos núcleos da base o que disponibiliza áreas corticais, como o córtex frontal, para a execução de tarefa novas ou menos aprendidas, que necessitam de direcionamento da atenção para sua execução de forma eficaz; isto diminuiria a interferência e favoreceria o desempenho de atividades concorrentes $^{27}$. No entanto, o intuito de desenvolver um treinamento em DT seria o de facilitar a aquisição e compartilhamento de controle atentivo específico para estas situaçôes, além da coordenaçáo de desempenho entre as tarefas, o que incrementaria a eficiência neural durante a execução de atividades simultâneas; consequentemente, esta forma de prática favoreceria também um incremento na manipulação de habilidades cognitivas ${ }^{19,28}$. Portanto, a automatização de uma das tarefas não necessariamente favoreceria o desempenho de DT. Deste modo, esperava-se que somente o GE apresentasse melhora no desempenho em DT, hipótese que foi confirmada somente na tarefa de atender ao telefone. Esta melhora pode estar associada à característica da tarefa. A combinação de uma tarefa de fluência verbal ao mesmo tempo em que se atende ao telefone pode ser considerada mais "ecológica", isto é, que faz parte do cotidiano do indivíduo, já que ao atender ao telefone requer o desempenho motor associado ao verbal.

Um estudo sobre o treinamento de idosos em tarefas de tempo de reação concorrentes verificou que mesmo após prática extensiva estes pacientes não foram capazes 
de executar as açóes de forma simultânea. Foi argumentado que apenas a prática da DT pode não ser eficiente para um melhor desempenho, porém se somada a estratégias como o conhecimento explícito das tarefas, orientaçóes quanto a priorização e realimentação adaptativa individualizada, poderia ser mais eficaz e facilitar o aprendizado ${ }^{29}$.

No presente estudo, ambos os grupos foram orientados a prestar atenção em ambas às tarefas, sem priorização, condição denominada de priorização fixa. Ainda não existem evidências se a priorização variável, quando os sujeitos são orientados a prestar mais atenção a uma das tarefas de forma variável a cada repetição, ou um treinamento com a priorização fixa é mais eficiente ${ }^{28}$.

É possível que pela característica complexa das atividades analisadas, a priorização variável com realimentação individualizada possa ser mais eficaz para o incremento do desempenho.

Os resultados demonstraram ainda que houve diferença de desempenho apenas em duas das tarefas discretas após o treinamento (atender ao telefone e pentear), sem diferença na de vestir um casaco a qual apresentou um grau de dificuldade mais elevado que as demais, pois exigia um componente postural já que foi executada em pé, estabilidade braço-mão para posicionar estes dois segmentos de forma precisa, além de destreza de dedos para manipulação de objetos pequenos. Além disso, esta tarefa é caracterizada como uma habilidade seriada, ou seja, composta por várias açóes conectadas em uma sequência determinada. Por fim, sabe-se que a bradicinesia afeta especialmente movimentos sequenciais, ainda mais em situação de DT e isto pode ter prejudicado a melhora do desempenho na tarefa de vestir o casaco.

As outras tarefas manuais (levar um copo a boca, atender telefone e pentear cabelo) podem ser consideradas mais simples, pois além de serem executadas na posição sentada, demandavam apenas a estabilidade braço-mão, destreza manual para manipular objetos relativamente grandes, além de caracterizadas como discretas, pois apresentavam um início e fim bem definidos, com breve duração $0^{30}$.

Em relação às tarefas em que não foi observado melhora no desempenho, acredita-se que um fator associado a este resultado pode ter sido o número insuficiente de repetições, já que PDP tem capacidade de aprender novas habilidades, porém necessitam de mais tempo de treinamento do que idosos da mesma faixa etária ${ }^{25}$.

Por outro lado, os pacientes avaliados estavam no estágio 1 e 2 da escala de HY, não apresentando ainda grande comprometimento motor; portanto, o desempenho atingido pelos sujeitos já na avaliação pode ter sido o máximo, justificando a não observação de incremento no desempenho após o treinamento. Deste modo, os próximos estudos poderiam compara os resultados com um grupo de idosos e também avaliar os efeitos do treinamento em pacientes com DP em estágios mais avançados da doença.

\section{CONCLUSÃO}

De modo geral, ambos os treinamentos contribuíram para a melhora da função de membro superior de pacientes com DP. No entanto, a condição de treinamento pode interferir na melhora do desempenho. A aprendizagem de tarefas de menor complexidade parece ser facilitada pela atençáo focada no desempenho, enquanto que tarefas que são cotidianamente realizadas em condições de divisão de atenção como o falar ao telefone, parece ser favorecida pelo treinamento em DT. Por outro lado, dependendo da tarefa, a divisão da atenção pode prejudicar a melhora no desempenho.

\section{REFERÊNCIAS}

1.Mcculloch KL. Attention and dual-task conditions: physical therapy implications for individuals with acquired brain injury. JNPT 2007;31:104-18.

http://dx.doi.org/10.1097/NPT.0b013e31814a6493

2.Yogev G, Hausdorff JM, Giladi N. The role of executive function and attention in gait. Mov Disord 2008;15:329-472. http://dx.doi.org/10.1002/ mds. 21720

3.Delong M, Wichmann T. Update on models of basal ganglia function and dysfunction. Parkinsonism Relat Dis 2009;15:237-40. http://dx.doi. org/10.1016/S1353-8020(09)70822-3

4.Cameron IG, Watanabe M, Pari G, Munhoz DP. Executive impairment in Parkinson's disease: response automaticity and task switching. Neuropsychol 2010;48:1948-57. http://dx.doi.org/10.1016/j.neuropsychologia.2010.03.015

5.Canning CG. The effect of directing attention during walking under dual-task conditions in Parkinson's disease. Parkinsonism Relat Dis 2005;11:95-9. http://dx.doi.org/10.1016/j.parkreldis.2004.09.006 6.Plotnik M, Giladi N, Hausdorf JM. Bilateral coordination of gait and Parkinson's disease: the effects of dual tasking. J Neurol Neurosurg Psychiatr 2009;80:347-50. http://dx.doi.org/10.1136/jnnp.2008.157362 
7.Plotnik M, Giladi N, Dagan Y, Hausdorf JM. Postural instability and fall risk in Parkinson's disease: impaired dual tasking, pacing, and bilateral coordination of gait during the ON medication state. Exp Brain Res 2010;210:529-38. http://dx.doi.org/10.1007/s00221-011-2551-0

8.Holmes JD, Jenkins ME, Johnson AM, Adams SG, Spaulding SJ. Dual-task interference: the effects of verbal cognitive tasks on upright postural stability in Parkinson's disease. Parkinsons Dis 2010;2010:696492. http://dx.doi. org/10.4061/2010/696492

9.Wu T, Hallet M. Neural correlates of dual task performance in patients with Parkinson's disease. J Neurol Neurosurg Psychiatr 2008;79:760-6. http:// dx.doi.org/10.1136/jnnp.2007.126599

10.Proud EL, Morris ME. Skilled hand dexterity in Parkinson's Disease: effects of adding a concurrent task. Arch Phys Med Rehabil 2010;91:794-9. http://dx.doi.org/10.1016/j.apmr.2010.01.008

11.Lu C, Bharmal A, Kiss ZH, Suchowersky O, Haffenden AM. Attention and reach-to-grasp moevements in Parkinson's disease. Exp Brain Res 2010;205:69-80. http://dx.doi.org/10.1007/s00221-010-2341-0

12.Benecke R, Rothwell JC, Dick JPR, Day BL, Marsden CD. Performance of simultaneous movements in patients with Parkinson's disease. Brain 1986;109:739-57. http://dx.doi.org/10.1093/brain/109.4.739

13.Brown RG, Jahanshahi M. An unusual enhancement of motor performance during bimanual movement in Parkinson's disease. J Neurol Neurosurg Psychiatr 1998;64:813-6.

14. Teixeira NB, Alouche SR. O desempenho da dupla tarefa na doença de Parkinson. Rev Bras Fisioter 2007;11:127-32.

15.Pradlam SD, Bambi RB, Carvell GE, Sparto PJ, Dellito A, Matsuoka Y. Assessment of fine motor control in individuals with Parkinson's disease using force tracking with a secondary cognitive task. JNPT 2010;34:32-40. http:// dx.doi.org/10.1097/NPT.0b013e3181d055a6

16.Berardelli A, Rothwell JC, Thompson PR, Hallett M. Pathophysiology of bradykinesia in Parkinson's disease. Brain 2001;124:2131-46. http://dx.doi. org/10.1093/brain/124.11.2131

17.Brauer SG, Broome A, Stone C, Clewett S, Herzig P. Simplest tasks have greatest dual task interference with balance in brain injured adults. Hum Mov Sci 2004;23:489-502. http://dx.doi.org/10.1016/j.humov.2004.08.020

18.Yogev-Seligmann G, Giladi N, Brozgol M, Hausdorff JM. A training program to improve gait while dual tasking in patients with Parkinson's disease: a pilot study. Arch Phys Med Rehabil 2012;93:176-81. $\quad$ http://dx.doi.org/10.1016/j.apmr.2011.06.005
19.Foreman KB, Sondrup S, Dromey C, Jarvis E, Nissen S, Dibble LE. The effects of practice on the concurrent performance of a speech and postural task in persons with Parkinson disease and healthy controls. Parkinsons Dis 2013;98:76-21. http://dx.doi.org/10.1155/2013/987621

20.Folstein MF, Folstein SE, Mchugh PR. Mini-mental state - practical method for grading cognitive state of patients for clinician. J Psychiat Res 1975;12: 189-98. http://dx.doi.org/10.1016/0022-3956(75)90026-6

21.Valle EA, Castro-Costa E, Firmo JOA, Uchoa E, Lima-CostaMF. Estudo de base populacional dos fatores associados ao desempenho no Mini Exame do Estado Mental entre idosos: Projeto Bambuí. Cad de Saúde Púb 2009;25:91826.

22.Paradela EMP, Lourenço RA, Veras RP. Validação da escala de depressão geriátrica em um ambulatório geral. Rev Saúde Púb 2005;39:918-23. http:// dx.doi.org/10.1590/S0034-89102005000600008

23.Guimarães CM, Brasil-Neto JP, Oliveira L, Valencia CEU. Desempenho motor em hemiparéticos após treino de relaxamento de membro superior não afetado. Rev Neurocienc 2010;19:439-503.

24.Seidler R. Neural correlates of motor learning, transfer of learning, and learning to learn. Exerc Sport Sci Rev 2010;38:3-9. http://dx.doi.org/10.1097/ IES.0b013e3181c5cce7

25.Stephan MA, Meier B, Zaugg SW, Kaelin-Lang A. Motor sequence learning performance in Parkinson's disease patients depends on the stage of disease. Brain Cogn 2011,75:135-40. http://dx.doi.org/10.1016/j.bandc.2010.10.015 26.Sydney S, Lang C. Using dual tasks to test immediate transfer of training between naturalistic movements: A proof-of-principle study. J Mot Behav 2012;44:313-27. http://dx.doi.org/10.1080/00222895.2012.708367

27.Goh HT, Sullivan KJ, Gordon J, Wulf G, Winstein CJ. Dual-task practice enhances motor learning: a preliminary investigation. Exp Brain Res 2012; 222: 201-10. http://dx.doi.org/10.1007/s00221-012-3206-5

28. Bherer L, Kramer AF, Peterson MS, Colcombe S, Erickson K, Becic E. Transfer effects in task-set cost and dual-task cost after dual task training in older and younger adults: further evidence for cognitive plasticity in attentional control in late adulthood. Exp Aging Res 2008;34:188-219. http://dx.doi. org/10.1080/03610730802070068

29. Maquestiaux F, Hartley AA, Bertsch J. Can Practice Overcome Age-Related differences in the Psychological Refractory Period Effect? Psychol Aging 2004;19:649-67. http://dx.doi.org/10.1037/0882-7974.19.4.649

30.Schmidt RA, Wrisberg CA. Motor learning and performance. 4rd ed.Champaign: Human Kinetics, 2008, 381p. 